\title{
Lageberichte sind oft qualitativ mangelhaft
}

Der Lagebericht soll einen Einblick zur aktuellen und künftigen wirtschaftlichen Situation des Unternehmens geben. In der Praxis lässt die Qualität der Lageberichterstattung jedoch oft zu wünschen übrig. Zu diesem Ergebnis kommt Martina Mühlbauer in ihrer Analyse „Die Qualität der Lageberichterstattung von DAX-Konzernen“.

Mühlbauer untersuchte für ihre empirische Analyse die Berichtsqualität des Teilberichts der Ertrags-, Finanz- und Vermögenslage und konzentrierte ihre Studie auf die Unternehmen des TecDAX. Als Qualitätsmaßstab dienten dabei die Anforderungen des Deutschen Rechnungslegungsstandards Nr. 15 (DRS 15). Das Ergebnis ist ernüchternd: Die Qualität ist in vielen Fällen unzureichend.

Im Einzelnen kommt Mühlbauer zu folgenden Erkenntnissen:

- Das durchschnittliche Qualitätsniveau der Lageberichterstattung ist mit einem Wert von 38,5 Prozent unzureichend.

- Den höchsten durchschnittlichen Erfüllungsgrad erreicht die Berichterstattung über die Gesamtaussage zur wirtschaftlichen Lage (53,7 Prozent).
- Gefolgt wird dieser Wert von der Berichterstattung zur Ertragslage (47,22 Prozent).

- Die Berichterstattung zur Finanzlage weist mit einem Qualitätswert von 32,47 Prozent ein unterdurchschnittliches Ergebnis auf, die Berichterstattung zur Vermögenslage mit deutlichem Abstand das schlechteste (18,77 Prozent).

Gerade für Unternehmen mit Kapitalmarktorientierung ist der Lagebericht ein wichtiges Aushängeschild, weil er z. B. neuen Investoren einen Überblick zur aktuellen wirtschaftlichen Lage und Prognosen zu den Entwicklungen bietet. Es bleibt abzuwarten, wann Unternehmen dieses Potenzial endlich ausschöpfen.

Mehr zum Thema:

Mühlbauer, M.: Die Qualität der Lageberichterstattung von DAXKonzernen - Empirische Analyse der Berichterstattung zur Ertrags-, Finanz- und Vermögenslage, Wiesbaden 2014, S. 404 - 412,

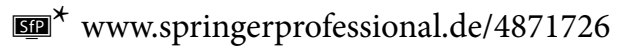

Sylvia Meier, Vera Treitschke, Wiesbaden

\section{Finanzierungsengpässe bedrohen Start-ups}

Viele Jungunternehmen versuchen, sich mit einer guten Geschäftsidee zu etablieren. Doch schon nach kurzer Zeit scheitern viele an der Finanzierung. Das „Start-up-Barometer Deutschland 2014“ der Wirtschaftsprüfungsgesellschaft Ernst \& Young spiegelt die aktuelle Situation von Jungunternehmen in Deutschland wider. Für die Studie wurden 151 Start-up-Unternehmen in Deutschland befragt, deren Gründung nicht mehr als acht Jahr zurückliegt.

Demnach blicken die meisten Start-ups zwar dank ihres Umsatzwachstums optimistisch in die Zukunft. Jedoch bereitet gerade die Finanzierung vielen Unternehmen Sorgen. Dabei ist entscheidend, in welchem Lebenszyklus sie sich gerade befinden. In der Seed-Phase fürchten laut der Studie über 90 Prozent der Start-ups Finanzierungsprobleme, in der Expansionsphase hingegen nur 22 Prozent.

Die Studie gibt weiter Aufschluss über die Finanzierungsmittel, die Start-ups nutzen. Es zeigt sich, dass je nach Unternehmensphase andere Finanzierungsmittel genutzt werden. So verwenden fast alle Unternehmen Eigenmittel und Cash- flow als Finanzierungsmittel. Gerade zu Beginn nehmen 15 Prozent der befragten Start-ups aber auch öffentliche Förderungen in Anspruch. In späteren Phasen der Unternehmensgründung nutzen einige Unternehmen Cashflow und Risikokapital als Finanzierungsmittel.

Interessant ist, dass der Umfrage zufolge Banken, Crowdfunding und Media for Equity in allen Phasen der Unternehmensgründung kaum eine Rolle spielen. Dabei ist der Bedarf nach Finanzierungsmöglichkeiten offenbar groß. Zwei Drittel der Jungunternehmen rechnen in den nächsten beiden Jahren mit weiterem Finanzierungsbedarf.

Mehr zum Thema:

Hahn, C.: Finanzierung und Besteuerung von Start-upUnternehmen, Wiesbaden 2014,

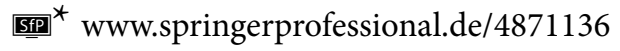

Sylvia Meier, Wiesbaden 